\title{
Plurality of Worlds: \\ Diversity of Exo-Earths and the \\ Implications for Life
}

\begin{abstract}
NATALie BATALHA ${ }^{1}$
${ }^{1} \mathrm{UCSC}$

NASA's Kepler spacecraft revolutionized our knowledge of planets around other stars, revealing thousands of planets in our galactic neighborhood. Using this treasure trove, we can explore fundamental questions about the processes that drive planetary diversity. We lean on exoplanet demographics to inform us about the domain of potentially habitable environments. NASA's TESS Mission is identifying nearby exoplanets whose atmospheres can be studied with the soon-to-be launched James Webb Space Telescope. The next decade calls for interdisciplinary studies to fully exploit these new discoveries and better understand how evolutionary history, geology, and climate conspire to create the conditions for life as we know it.
\end{abstract}

